\title{
AVERROES Y TOMÁS DE AQUINO SOBRE EL CONCEPTO DE CIENCIA NATURAL
}

\author{
Celina A. Lértora Mendoza
}

\begin{abstract}
RESUMO - Al comienzo del libro I de la Physica, Aristóteles plantea la pregunta por la materia y el sujeto de la ciencia natural. Esta cuestión se refiere al concepto de la ciencia cuya indagación inicia. La ciencia natural (philosophia naturalis) ha sido en lo sucesivo objeto de indagaciones específicas, sobre todo en función del comentario al original aristotélico. Averroes ${ }^{1}$ dedica al tema un conciso y a la vez comprehensivo Proemio. Tomás de Aquino, ${ }^{2}$ en cambio, y al contrario de otros casos, omite un proemio metodológico, si bien el tema en sí se desarrolla al hilo del comentario del texto del Libro I. El objetivo de este trabajo es analizar las aproximaciones y las diferencias entre la lección I del Comentario al Libro I del Aquinate (donde trata el método de la philosophia naturalis) y la exégesis de Averroes en su Proemio y la Summa Prima, a fin de establecer las relaciones entre ambos.
\end{abstract}

PALAVRAS-CHAVE - Aristóteles. Tomás de Aquino. Averroes. Ciencia natural. Metodología física.
ABSTRACT - At the beginning of the Book 1 of the Physica, Aristotle sets the question on the matter and subject of natural science. This issue refers to the concept of the science, which he starts bringing up. Natural Science (philosophia naturalis) has, since then, been especially enquired into, above all in terms of the original Aristotle's commentary. Averroes dedicates a concise and, at the same time comprehensive Proem on the subject. Thomas Aquinas, on the contrary, and in opposition to other cases, omits a methodological proem, although the subject itself develops on the brim of the commentaries on the text of Book 1. The aim of this work is to analyze the approximation and differences between the Lesson 1 of the Commentaries to the Book 1 of the Aquinate (where the philosophia naturalis method is dealt with) and the exegesis of Averroes in his Proem and the Summa Prima, in order to establish the relationship between both.

KEY WORDS - Aristotle. Thomas Aquinas. Averroes. Natural science. Physical methodology.

\section{La presentación de Averroes}

En primer lugar, Averroes justifica el comentario completo que inicia recordando que ninguno de los expositores por él conocidos había realizado una glosa completa. Con esto adelanta, indirectamente, que para tal cometido no tiene mo-

Conicet- Buenos Aires.

Cito por Aristotelis Opera cum Averrois Commentariis, Venetiis, apud Junctas 1562-1574, T. IV: Aristotelis de Physico Auditu libri octo cum Averrois cordubensis veriis in eosdem commentariis.

2 Cito por S. Thomae Aquinatis In Octo Libros Physicorum Aristotelis Expositio, cura et studio P. M. Maggiòlo O. P, Turín-Romae, Marietti, 1965.

\begin{tabular}{|l|l|l|l|l|l|}
\hline VERITAS & Porto Alegre & v. 52 & n. 3 & Setembro 2007 & p. 149-158 \\
\hline
\end{tabular}


delos; por otra parte reafirma incidentalmente ${ }^{3}$ su método de comentario paso a paso y su adhesión al método de los glosadores, según el cual debe comenzarse por explicitar ocho puntos: 1. la intención de la obra; 2. su utilidad; 3. su orden; 4. su división; 5. su estructura; 6. el desarrollo de la doctrina; 7. el título de la obra; 8. el autor. Obviamente de todos estos puntos el primero es decisivo como clave hermenéutica y en cierto modo condiciona a los siguientes. La intentio authoris se ratifica como el primero y principal de los criterios exegéticos y al que se subordina cualquier otro. La mayor parte de las discusiones y divergencias entre el Cordobés y otros expositores radica precisamente en una discrepancia sobre este punto o en el rechazo por su parte de interpretaciones que no coincidan con lo que él considera intención cierta de Aristóteles en esta obra.

Ahora bien, la intención del autor como criterio exegético principal -si no único- en caso de duda, todavía debe ser precisada, al menos en tres aspectos: 1. en cuanto a su amplitud en relación a la obra total, 2. en cuanto al grado de adhesión que dicho autor quiso darle a una teoría; 3. de qué modo el intérprete accederá a tal "intentio" y por lo tanto cuál es el método científicamente válido de componer una "interpretación auténtica".

Con respecto al primer punto, Averroes, siguiendo en esto a sus antecesores, la considera específica y exclusiva de la obra, es decir, cada obra adquiere su unidad precisamente de su objetivo o "intentio"; por lo tanto, el propósito general o - podríamos añadir - la concepción básica del Filósofo servirá como criterio accesorio, de concordancia, una vez establecida la intención de una obra en particular, pero no a la inversa, es decir, el objetivo concreto de una obra no puede derivarse hermenéuticamente de lo que sabemos sobre la intentio general ${ }^{4}$ o las propias de otras obras.

Con respecto al segundo punto, el grado de adhesión otorgado a una exposición, la cuestión parece aún más compleja. De hecho, en este punto se producen las mayores discrepancias interpretativas entre dos exegetas que, como Averroes y Tomás de Aquino, coinciden metodológicamente en lo esencial. ${ }^{5}$ En efecto, el grado de adhesión o el alcance de compromiso, en principio, y para quienes, como el Cordobés y el Aquinate, se inclinan decididamente por el método literalista, sólo puede ser establecido con un grado razonable de confiabilidad, a partir de tres

3 Al indicar la ausencia de comentarios completos señala: "quia a nullo expositorum aedita est glossa completa super singulis verborum Aristotelis..." (subrayado mío), ob. cit, f. 1 B.

4 El tema de si puede - y como - establecerse una intentio general para el corpus filosófico de un autor, partiendo del criterio averroísta de delimitar los objetivos específicos, sin duda es muy interesante, pero lo omitiré aquí. Por lo que hace al mismo Averroes, él acepta para su propia teoría natural el criterio más amplio de adoptar y adaptar los principios fundamentales de todo saber, que según el análisis de M. Cruz Hernández, se resumen en dos afirmaciones: 1. todo saber está fundado en principios reales; 2 . los principios de la ciencia se fundamenta en verdades universales que permiten pensar universalmente (cf. "Los principios fundamentales de la filosofía de la naturaleza de Averroes", en La filosofia della natura nel Medioevo, Milano, 1964, pp. 178-183).

5 Me he ocupado de la divergencia exegética de este tipo en "Verdad, apodicticidad y argumentación. Algunos casos de Santo Tomás, Aristóteles y Averroes presentes", Veritas 46, n. 3, 2001: 417430. 
indicios ciertos: 1. el texto explícito del autor, indicando en qué sentido entiende su exposición; 2. el contexto argumentativo inmediato en que se enuncia; 3. el contexto general de la obra y/o de la doctrina del Filósofo. Dejando de lado el primer supuesto (inaplicable por hipótesis), nos quedan los otros dos. Y aun cuando ambos contextos fuesen claros (lo que no siempre sucede) ¿qué pasa si no coinciden? ¿a cuál dar prioridad? A mi juicio, en este punto hay una divergencia interpretativa clara entre Averroes y Tomás. Para el primero, siempre prevalece el contexto inmediato, no sólo en cuanto al significado de los términos o conceptos empleados, sino y sobre todo en cuanto al carácter lógico de la argumentación, el que fija el estatuto científico del teorema en cuestión (es decir, si es apodíctico o no, qué modalidad -necesario-probable- le corresponde, etc.). Tomás, en cambio, no sigue siempre este criterio, sino que a veces recurre a contextos más amplios, dentro de la misma obra o incluso fuera de ella. Dado que en ambos supuestos se respeta el criterio intrasistemático de la búsqueda de la intentio authoris, resulta difícil establecer cuál de los dos debe prevalecer. De todos modos, en el caso en estudio, es decir, analizando el proceso de recepción de la doctrina aristotélica en Tomás de Aquino a través de Averroes, ${ }^{6}$ es claro que la opción tomasiana funciona como un veto (metodológicamente correcto en la opción literalista) cuando el resultado exegético averroísta no le resultaba aceptable. Las razones por las cuales una exégesis puntual averroísta no es aceptable se vinculan, en mi concepto, con el nexo lógico o sistemático que puedan tener con otras exégesis puntuales importantes y rechazables por otras razones (por ejemplo, por contrariar el dogma cristiano). ${ }^{7}$

Sobre el tercer punto, es decir, el método para detectar la intentio authoris no hay discrepancia entre ambos comentadores, que se decantan claramente por el método literalista. De modo que las palabras de Aristóteles prevalecen sobre cualquier otro criterio, salvo que - y esto es importante - estas palabras contradigan abiertamente otras. En este caso, la conclusión de Averroes es que los dos textos no pueden pertenecer al mismo autor, es decir, no se supone una contradicción subjetiva o inconsistencia de pensamiento, sino que uno de los dos pasajes debe ser espúreo. Para determinar cuál de los dos lo es, debemos recurrir a una ampliación de la exégesis contextual inmediata, y este es posiblemente el único caso, aunque muy relevante, en que el Cordobés se aparta de su estricto criterio del "paso a paso", con resultados muy aproximados a los que mucho después estable-

\footnotetext{
La influencia general de la exégesis averroísta sobre la latina, especialmente la tomasiana, no ha sido cuestionada desde el trabajo pionero y decisivo del P. De Vaux ("Le premier entrée d'Averroes chez les latins", Rev. de Sciences Philosophiques et Théologiques, 22, 1933: 194-243); sin embargo, los préstamos concretos no han sido analizado por los estudiosos del Aquinate, como he tenido ocasión de señalar en los artículos citados en este trabajo.

Asumo que esta hipótesis debe ser analizada en cada caso concreto, y yo misma lo hago, para uno de ellos, en el artículo citado y en "Tomás de Aquino frente a Averroes. El Comentario a la Física de Aristóteles, libro VII: prueba del primer motor", Luis A. De Boni, Roberto H. Pich (orgs.) A recepção do pensamento greco-romano, árabe e judaico pelo Occidente Medieval; Porto Alegre, Edipucrs, 2003: 421-437.
} 
cería una crítica histórico filológica en posesión de recursos teóricos de los que él carecía.

Pasemos a los ocho puntos enunciados en el proemio.

La intentio del libro coincide con la del corpus físico en general, de la cual éste es la primera parte: conocer las causas de las sustancias sensibles y de sus accidentes, $^{8}$ considerando sustancias sensibles o naturales a las que tienen en sí el principio del movimiento y el reposo. Esto podría considerarse el objetivo general. El objetivo específico es el conocimiento de las causas comunes a todas las cosas naturales (materia, forma, fin y agente) y la determinación de las causas del conocimiento de ellas, es decir, cuál es el principio de inteligibilidad o de cognoscibilidad de las cosas naturales. Volverá luego sobre el problema específico del método, en la Suma Primera del comentario al Libro Primero. En síntesis, importa señalar que si bien Averroes se atiene al criterio del objetivo o tema específico (intentio), no lo desvincula del conjunto de las obras naturales por lo cual se producirá una conexión normalizada en la exégesis de todo el corpus físico. La unidad de dicho corpus le viene precisamente de tener la misma intentio, por lo cual el exegeta puede ampliar el contexto interpretativo homogéneamente a todo este corpus, pero para ampliarlo a otras obras no físicas, tal ampliación debería justificarse por otros argumentos.

La utilidad se conecta con la utilidad general de la ciencia especulativa, que es la perfección intelectiva del hombre. ${ }^{9}$ En esto no hace sino seguir al mismo

\begin{abstract}
Sobre la relación de este concepto de sustancia averroísta y el de materia con el cual se vincula estrechamente (en filosofía natural) v. A. Pérez Estévez, "Materia y sustancia en Averroes", Ernildo Stein (org.) A cidade de Deus e a cidade dos homens de Agostinho a Vico. Porto Alegre, Edipucrs, 2004: 503-526.

"Et declaratum est in scientia consyderante in operationibus voluntariis quod esse hominis secundum ultimam perfectionem ipsius, et substantia eius perfecta est ipsum esse perfectum per scientiam speculativam; et ista dipositio est sibi foelicitas, et sempiterna vita" (f. 1v H). En el proemio Averroes dedica varios párrafos a este tema de la virtud especulativa, que en su conjunto constituyen casi la mitad del texto. Aunque no es tema del presente trabajo, quisiera señalar que este pasaje, explícitamente apoyado en Alejandro, se orienta a fundamentar la tesis de que el hombre sabio es feliz y que la sabiduría teórica (la ciencia) es la base y el sustento de todas las virtudes morales; en especial menciona la justicia, la temperancia, la fortaleza, la magnanimidad, la liberalidad, la verdad, la fe y la mansedumbre. No me parece casual la referencia a Alejandro, ya que de este modo justifica (no a título propio sino como mención de otro exegeta célebre) la constitución racional de una moral laica. Es legítimo sospechar que Averroes hace suyo el pasaje de Alejandro que menciona, incluso poniendo a Sócrates como ejemplo, y también que lo hace como un modo de exponer su propio pensamiento escapando a la censura: "Quia, cum noverit sapiens parvitatem vitae suae, et perpetuitatem sempiternam, et motum continuum, et quod proportio suae vitae ad tempus sempiternum est sicut puncti ad lineam, vel sicut minimum finitorum ad infinitum, non procuravit propriam vitam, et tunc erit fortis ex necesítate. Et similiter, cum noverit sapiens quod mors est ex necessitate materiae, et proprie cum habet perfectionem humanam, et dubitaverit quod a perfectione expolietur, tunc non est mirum, si aliquando eligit mortem prae vita; sicut fecit Socrates cum Atheniensibus..." (f. 2 A). En el último de los párrafos dedicados a este asunto, Averroes hace una referencia negativa a aquellos que, buscando la perfección por esta vía, consideran que no deben ocuparse de los asuntos políticos ("non debent esse pars civitatis") y consciente de que se está apartando del tema, concluye "Et etiam exivimus a nostra intentione, ad quam revertimur" (f. 3 B). No es difícil ver aquí una cierta apología de la moralidad secular, y quizá el silencio de Tomás de Aquino acerca de la "utilidad" de la filosofía natural indicaría que no puede compartir este punto de
\end{abstract}


Aristóteles, y aunque el tema daría lugar a una interesante discusión filosófica, excede el propósito de este trabajo.

El orden del libro, continúa, es el que le corresponde por ser el primero de la serie de los naturales, de tal modo que como las cosas naturales se diversifican según sus diferentes naturas, teniendo sin embargo algo en común todas ellas, corresponde dividir el corpus físico en dos partes: en la primera se analizan las propiedades comunes a todas las cosas naturales, y en la segunda lo propio de cada uno de sus géneros, de tal modo que esta segunda parte se divide, a su vez, conforme a dichos géneros. Vemos entonces que en la interpretación averroísta, el corpus natural aristotélico adquiere una estrecha trabazón, de tal modo que los principios de la Física serán aplicados, como postulados generales, a todas las obras físicas, procediendo en consecuencia una exégesis de ellas fundada y eventualmente deducida de la Física, aunque, por supuesto, no a la inversa. El resultado de este criterio es doble: por una parte, el fortalecimiento de todo el sistema físico, incluyendo obras que en otra perspectiva podrían considerarse ligadas a otros principios no expuestos en la Física, como el De Caelo, en un extremo, o el De partibus animalibus, en el outro. ${ }^{10}$ Por otra, lo deslinda claramente no sólo de la filosofía segunda o práctica (lo que es obvio) sino también de otras partes de la filosofía sistemática, en especial de la Metafísica. Sin embargo, el mismo Averroes postula a la Física como un elemento decisivo para la comprensión de la Metafísica. Esto se explica, en mi criterio, si suponemos que Averroes, aun cuando comparte la postura deductivista de la ciencia, acepta la construcción de la Metafísica a partir de una ampliación (fundamentada) de la Física pero no a la inversa. En otros términos, que sostiene una versión de la llamada "teoría de los tres grados de abstracción".

La división de la obra que asume es en primer término la de las ocho partes o libros, añadiendo que a su vez cada uno se divide en secciones mayores y menores o subsecciones. Pero como esto no está marcado por los editores, estas divisiones son propuestas por él mismo, constituyendo las "Sumas" en que se divide cada libro y los parágrafos o subsecciones de cada ellas. Averroes no presenta ninguna justificación, en este proemio, de su división del texto para cada libro. Sin embargo, puede deducirse cuál ha sido su criterio al observar sus propias divisiones. Cada una de ellas presenta - al menos en la interpretación de Averroes - el desarrollo completo de un tema, y dentro de cada "suma", los parágrafos avanzan conforme a los pasos completos de un razonamiento parcial.

vista. La mención averroísta de Alejandro en este punto es particularmente significativa, si tenemos en cuenta que no era un comentador grato al Cordobés, por su materialismo y su noética simplista (Cf. B. C. Bazán "La noética de Averroes", Philosophia n. 38, 1972, p. 21).

10 Obsérvese que Averroes escribe en una época que consideraba "ciencia normal" astronómica el sistema ptolemaico y que las investigaciones post aristotélicas sobre biología presentaban diferencias sustanciales con estos trabajos que, por otra parte, nunca fueron considerados por su autor como estrictamente "científicos", en sentido fuerte, o sea, en el sentido exigido en los Posteriores Analíticos, ya que él mismo había establecido para el conjunto de investigaciones empíricas la categoría pre-científica (pre-científica, obviamente, en relación a su propia concepción "fuerte" de ciencia, o sea, como conocimiento exclusivamente derivativo) de la taxonomía. 
La estructura (proportio) se relaciona al conjunto de la ciencia natural como la parte al todo, considerando que el "todo" es el conjunto de la ciencia especulativa que incluye la matemática y la metafísica. Tenemos aquí, de nuevo, una versión propia de la "teoría de los grados de abstracción", aunque no en sentido gnoseológico (como en la versión tomista) ni en relación al uso. En este sentido la ciencia natural y la teología se contraponen a la matemática. ${ }^{11}$

La estructura de este libro, por otra parte, se relaciona al conjunto de la ciencia natural (subconjunto de la ciencia especulativa), no como la parte al todo sino como los "elementos" (fundamentos) de las otras, porque en ella se explican los principios y raíces de todas las verdades que puedan establecerse en las demás. Nuevamente vemos que el criterio de Averroes es apuntalar la unidad de la ciencia física, de tal modo que en las demás obras esté implícito el contenido de ésta, ya que sin ella no podrían avanzar en sus propios campos de investigación.

El desarrollo de la doctrina incluye los métodos usuales de esta ciencia que, según Averroes, son propios a todas las disciplinas, conforme lo postula el propio Aristóteles, es decir, tres formas de demostración: por el signo, por la causa y demostración absoluta (es decir, absolutamente universal), señalando que las dos primeras son más usadas en la ciencia natural, aunque también a veces se usa la tercera. Anuncia también que el Estagirita usa - como modos adquisitivos de conocimiento científico - la definición, la división, la inducción y el ejemplo, advirtiendo que se sigue la justificación dada en los Posteriores Analíticos, aunque el ejemplo y la inducción (que entre sí son diferentes) son propios de la Retórica y la Dialéctica ${ }^{12}$ Con esto quiere significar que en la construcción física hay elementos dialécticos que deben ser claramente explicitados, pero que ellos no obstan a la cientificidad del corpus. Ahora bien, esto será así, en la medida en que la inducción y el ejemplo no sean parte determinante de una construcción teórica natural, lo cual precisamente deberá ser mostrado por el exegeta en cada caso. De ahí el cuidado que se aprecia a lo largo del comentario, en justificar el tipo de argumentación y el papel que la inducción y el ejemplo juegan en ella.

El nombre que retiene como original y más propio (auditus naturalis) no lo vincula, como luego se hizo, al hecho de provenir de "lecciones orales" sino que lo toma como equivalente a "discurso natural" (sermo naturalis) con el cual se debería denominar a todo el corpus; de modo que, aceptando esto, concluye que se ha producido una figura retórica de apropiación del nombre del todo para darlo a una parte, lo que se justifica, nos dice, porque es la más importante.

Finalmente, el autor, sin ninguna duda es Aristóteles, "el más sabio de los griegos", que compuso una obra científica completa, ya que abarca las tres partes de la: la Lógica, la Física y la Metafísica, en lo cual ha superado a sus antecesores. La

\footnotetext{
11 "Quia scientiae speculativae sunt duobus modis: unus est propter exercitium, sicut Mathematica; alius propter perfectionem, propter quam ipsum exercitium est. Et est scientia Naturalis, et Divina" (f. 4 A).

12 Digamos que el uso del "ejemplo" en este sentido no equivale al principio de experiencia, que es aceptado por Averroes como elemento falsador de una teoría.
} 
admiración de Averroes por Aristóteles le lleva a afirmar algo que, pudiendo considerarse un recurso literario, termina siendo un principio exegético: que el Estagirita no cometió ningún error, o al menos que desde que escribió su obra hasta nosotros no se le ha podido encontrar ninguno. ${ }^{13}$ Creo que con esto quiere decir que el sistema de Aristóteles es absolutamente consistente. Partiendo de esta premisa (implícita en su alabanza) es claro que su comentario, a diferencia de otros, tenderá en todos los casos a justificarlo, dando la interpretación que fortalezca más la consistencia. De no aceptarse este criterio, es evidente que la elección entre dos interpretaciones plausibles puede conducir a resultados diferentes.

\section{La presentación de Tomás de Aquino}

El texto de Tomás, a diferencia del de Averroes, no tiene ningún prólogo ni presentación y se inicia directamente con el comentario al libro primero. Sin embargo, los ocho puntos introductorios mencionados se encuentran implícita o explícitamente en la Lección Primera, a partir de la cual procuraré reconstruir la respuesta tomasiana. ${ }^{14}$

La intentio authoris se relaciona pero no se identifica con lo que Tomás llama su materia et subjectum. Es decir, no habla de la intención o propósito personal de Aristóteles, sino que presenta la cuestión como algo objetivo, en el sentido de que hay una materia o sujeto (tema propio y específico) de la ciencia natural que es lo que se investiga en ella, y aunque parece claro que esa ha sido la intención de Aristóteles, en cuanto tal, dicha intención no constituye un elemento decisivo para la fijación de los criterios hermenéuticos. Creo que haber omitido toda referencia inicial a la intentio authoris no es casual, sino que responde a la opción de no usar este criterio en forma exclusiva y tal vez ni siquiera prevalente. Tampoco es que Tomás rechace el criterio, pues en muchos pasajes explícitamente indica que se atiene a la "intención de Aristóteles" (incluso no compartiendo sus resultados). Esta separación de la intentio authoris tiene, a mi modo de ver, un efecto paradójico: aunque aparentemente reste "autoridad" al Estagirita, confiere al contenido del texto un valor objetivo (por así decirlo) mucho más fuerte; en otros términos, la ciencia natural es esto, no porque esa fuera la intención de Aristóteles sino porque es así. La eliminación de la historicidad del texto (subrayada por Averroes en especial debido a su admiración por el Estagirita) tiene el efecto de petrificar los contenidos. ${ }^{15}$

${ }_{13}$ "Complevit quia nullus eorum, qui secuti sunt eum usque ad hoc tempus, quod est mille et quingentorum annorum, nihil addidit, nec invenit in eius verbis errorem alicuis quantitatis. Et talem virtutem esse in individuo uno miraculosum, et extraneum existit. Et haec dispositio, cum in uno homine reperitur, dignus esse divinus magis, quam humanus" (f. 5 A-B).

${ }^{14}$ Es decir, voy a utilizar el texto de la Lección I del Libro I, no para compararla con los pasajes respectivos de Averroes en cuanto al contenido, sino sólo para reconstruir lo que serían los prolegómenos a la ciencia natural: su concepto, importancia, división y método.

${ }_{15}$ Incidentalmente señalo que los discípulos de Tomás hicieron lo mismo con sus propios textos. Hoy no estaríamos de acuerdo con este criterio, y somos más propensos a pensar que la "ciencia natu- 
La utilidad tampoco es un punto explícito pero puede colegirse que Tomás estaría de acuerdo con Averroes en sus dos puntos, ya que la perfección de la ciencia especulativa y su carácter de fundamento de la filosofía práctica, es doctrina propia. En cambio la preeminencia absoluta (para todo saber, en general) que otorga Averroees concede a la filosofía no es doctrina tomasiana y en este punto no habría acuerdo.

El orden tampoco se enuncia en forma explícita; obviamente sigue la división en libros, pero la división redaccional en lecciones no responde a la división lógica, para la cual Tomás tiene un criterio propio, diferente del averroísta: él usa el principio analítico, dividiendo el texto siempre en forma bimembre o booleana (salvo casos en los cuales es evidentemente inaplicable). De este modo, a veces forzado, se incluyen todos los temas a los que Averroes concede entidad teórica propia, pero sin su conexión derivativa, ${ }^{16}$ lo que, por otra parte, es el criterio general de Tomás en todos sus comentarios a Aristóteles. Haya sido o no una elección motivada en este sentido determinado, de hecho produce el efecto de separar y/o aflojar (debilitar) los nexos lógicos entre los teoremas del sistema. De ese modo, es posible (metodológicamente hablando) separar uno de ellos sin causar un daño irreparable al conjunto. Está claro que una propuesta hermenéutica encaminada a "cristianizar a Aristóteles", eliminando de su sistema todo lo que no fuese aceptable para el dogma, pero sin producir en él inconsistencias (al menos visibles) que lo tornaran inservible para los fines que la intelectualidad cristiana latina se proponía, sólo podía hacerse de este modo.

Sobre la estructura sí tenemos una posición explícita aunque no como respuesta a esta pregunta, y es la exposición de la teoría de los grados de abstracción enunciada en el segundo párrafo de la lección, suficientemente conocida. En cambio, nada se dice aquí de los métodos usados por el Estagirita, sino que se los identifica en cada caso y cuando la mención es necesaria para dilucidar el carácter y el tipo de argumento.

En cuanto al nombre, Tomás asume el más corriente en ese momento (liber Physicorum) al cual considera el primero de la ciencia natural. Y acerca de ella, a continuación, trata su estatuto epistemológico con la teoría de los tres grados ya mencionada. Probablemente esta diferencia entre ambos comentarios no es sustancial, pero quiero señalar algo: para Averroes la Física no es una parte del conjunto (homogénea a las otras) sino el fundamento, algo así como la teoría general; Tomás omite esta explicitación; sin embargo él mismo la usa como fundamento al

ral" es un constructo histórico en el cual la participación de Aristóteles fue decisiva, aunque debemos ser cautos al atribuir a Averroes este punto de vista contemporáneo.

16 Me ocupé de este tema en mi trabajo "El Comentario de Santo Tomás a la Física: la división del texto aristotélico", Sapientia 37, n. 212, 2002: 393-440. He señalado en varias oportunidades, a aquí lo reafirmo a partir de la inexistencia de una justificación del método, que Tomás sigue en realidad parcialmente la propia metodología científica del Estagirita, ya que usa sólo el método analítico y no el derivativo. Grosseteste, por ejemplo, en cambio, divide el texto aristotélico según lo que él considera conclusiones científicas independientes (teoremas), y en esto está más en la línea de Averroes, aunque la prolijidad del comentario paso a paso de éste desdibuja el intento de mostrar el entramado derivativo del sistema aristotélico. 
comentar otras obras del corpus, lo que implica que pensaba lo mismo que Averroes. Pero no se compromete con la afirmación y esto, teniendo en cuenta el resultado de la exégesis averroísta (en relación a su propio proyecto hermenéutico), me parece significativo.

Finalmente, el nombre del autor no es tematizado en absoluto, se da por supuesto y no hay ninguna referencia comparable con la admiración exclamativa que cierra el proemio averroísta. Teniendo en cuenta la situación que Tomás enfrentaba al tiempo de la composición del comentario, esta sobriedad está plenamente justificada. Su real admiración por Aristóteles radica más en su propia asimilación de lo esencial de la propuesta filosófica del Estagirita, incluso contra la más fuerte corriente opositora que debió enfrentar (en vida y después de muerto).

\section{Breve consideración final}

Los dos comentarios que tenemos en vista han sido considerados los mejores en su tipo (exhaustivos y sistemáticos) y la preferencia por uno u otro y las discusiones posteriores sobre cuál de ellos es "mejor" no pueden sino pasar por consideraciones vinculadas a los objetivos de los mismos. Aclarado el objetivo, me parece, se explican bien todas estas diferencias que he mencionado y otras que detectan diversas investigaciones.

Tal vez la diferencia decisiva entre ambas es que Averroes se propone un comentario de tipo histórico-crítico ${ }^{17}$ y Tomás un comentario al servicio de un proyecto filosófico propio. Que difícilmente puedan conjugarse ambas motivaciones lo demuestra este caso, en el cual hay diferencias insalvables y que resulta dudoso que puedan ser dirimidas por un procedimiento consensuado por ambas partes. Y esto por dos motivos. El primero, porque la intentio authoris histórica, además de ser en sí misma problemática en muchos casos (incluso porque a su vez depende de asunciones hermenéuticas históricas que pueden no ser compartidas) siempre puede ser "superada" con dos recursos: 1. considerar el principio hipotético de que "si Aristóteles hubiera conocido tal verdad (que ahora conocemos) había modificado su teoría en tal sentido". Es claro que este condicional contrafáctico es lógicamente incorrecto e históricamente al menos dudoso, pero de hecho puede usarse ideológica o exhortativamente para buscar interpretaciones usables; 2. el principio de "explicitación de lo implícito", que asume que todas (o al menos las más importantes) deducciones de una teoría pueden ser adscritas al autor, si no por el principio hipotético anteriormente mencionado, sí como "reconstrucción racional" de su propio pensamiento. Este punto de vista, aunque más correcto desde el punto

\footnotetext{
Esto no significa que Averroes se desinterese del resultado exegético en relación a sus opciones filosóficas. Como ha señalado Cruz Hernández, hay tres hitos en suposición: la ruptura sistemática de la síntesis neoplatónica, el énfasis en el naturalismo (incluyendo un amplio recurso a lo empírico) y la separación entre ciencia y fe (Cf. Anû-1-Walîd Mamad ibn Rushd (Averroes): Vida, obra, pensamiento, influencia, Córdoba, $2^{a}$ ed.1997, reiterando ideas expuestas en "Los principios fundamentales..." cit.). Es claro que el resultado de sus comentarios al corpus físico aristotélico fortalece la segunda de las líneas propias mencionadas.
} 
de vista lógico-metodológico, tiene el inconveniente de la desmesura del resultado, que razonablemente no puede adscribirse a un ser humano, cuya capacidad de tener cierta conciencia de lo implícito de su pensamiento es limitada. Y por otra parte, la deducción de teoremas a partir de otros más generales (incluso en una disciplina formal y estricta como la lógica o la matemática) no siempre se hace de un solo modo, por lo cual no podemos saber cuál de las vías posibles hubiera tomado el autor del pasado; de modo que en realidad hemos de considerar estos avances como propios de sus seguidores o de su escuela, pero no del fundador. ${ }^{18}$

El segundo motivo - y el más decisivo, a mi entender - por el cual no parece posible un consenso entre los dos criterios, es que la hermenéutica instrumental depende esencialmente del proyecto filosófico al cual sirve, y si los proyectos que quieren servirse de una exégesis de este tipo son incompatibles, no hay modo de compatibilizarla.

Así las cosas, tal vez debamos resignarnos a continuar teniendo dos comentarios excelentes que visualizan un Aristóteles en parte igual y en parte diverso, sin que la cuestión del "verdadero Aristóteles" - aun cuando logre resolverse con criterios histórico-crítico-filológicos - quede resuelta como "cuestión filosófica". Lo cual, lejos de parecerme lamentable, me resulta altamente estimulante.

${ }_{18}$ Incluso la divergencia entre los resultados globales e incluso más o menos consensuados de una escuela, o también las divergencias insalvables dentro de la misma, ha llevado a distinguir la doctrina histórica del fundador y la doctrina de la escuela, y así se habla de "doctrina tomasiana" y "doctrina tomista" (o, para un autor más reciente incluso "doctrina marxiana" y "doctrina marxista" con lo cual volvemos la punto de partida de la dificultad histórico.-crítica. 